\title{
Identification and Control of Distillation Process using Partial Least Squares based Artificial Neural Network
}

\author{
Seshu Kumar Damarla \\ Department of Chemical Engineering, \\ Maulana Azad National Institute of Technology \\ Bhopal, India.
}

\author{
Madhusree Kundu \\ Department of Chemical Engineering, \\ National Institute of Technology Rourkela, \\ Rourkela, India.
}

\begin{abstract}
Partial least squares technique has been in use for identification of the dynamics \& control for multivariable distillation process. Discrete input-output time series data $(X-Y)$ were generated by exciting non-linear process models with pseudo random binary signals. Signal to noise ratio was set to 10 by adding white noise to the data. The ARX models as well FIR models in combination with least squares technique were used to build up dynamic inner relations among the scores of the time series data $(X-Y)$, which logically built up the framework for PLS based process controllers. In this work, process dynamics was also identified in latent subspace using neural networks. The inverse dynamics of the latent variable based NN process acted as inverse neural controller (DINN). Distillation process without any decoupler could be controlled by a series of NN-SISO controllers
\end{abstract}

\section{General Terms}

Process Identification \& control, Statistical Process Control

\section{Keywords}

Partial Least Squares, NNPLS, DINN.

\section{INTRODUCTION}

Partial least square is one of the important multivariable statistical process control (MVSPC) techniques to find the latent variables from the measured data by capturing the largest variance in the data and achieves the maximum correlation between the predictor $(X)$ variables and response $(Y)$ variables. First proposed by Wold PLS has been successfully applied in diverse fields including process monitoring, identification of process dynamics \& control and it deals with noisy and highly correlated data, quite often, only with a limited number of observations available [1]. A tutorial description along with some examples on the PLS model was provided by Geladi Kowalaski [2]. When dealing with nonlinear systems, the underlying nonlinear relationship between predictor variables $(X)$ and response variables $(Y)$ can be approximated by quadratic PLS (QPLS) or splines. Sometimes it may not function well when the non-linearities cannot be described by quadratic relationship. Qin and McAvoy suggested a new approach to replace the inner model by neural network model followed by the focused R\& D activities taken up by several other researchers like Wilson et al. (1997); Holcomb \& Morari (1992); Malthouse et al. (1997); Zhao et al. (2006); Lee et al. (2006) [3-9]. This approach of NNPLS employs the neural network as inner model keeping the outer mapping framework as linear PLS algorithm. The conventional PLS is suitable for modeling time independent or steady state processes. Kaspar and Ray (1993) developed dynamic extension of the PLS models by filtering the process inputs and subsequent application of the standard PLS algorithm and demonstrated their approach for identification \& control problem using linear models [10]. Lakshminarayanan, 1997 proposed the ARX/Hammerstein model as the modified PLS inner relation and used successfully in identifying dynamic models and proposition of PLS based feed forward and feedback controllers [11]. For modeling dynamic process, the input data matrix $(X)$ is augmented either with large number of lagged input variables (called finite impulse response (FIR) model) or including lagged input and output variables (called auto regressive model with exogenous input, ARX). By combining the PLS with inner ARX / FIR model structure, dynamic processes can be modeled apart from using NN. One of the earlier approaches of multivariable control had been the decoupling control to reduce the loop interactions. The decoupler combined multivariable processes were used to create as series of NN-SISO controllers and tuned independently without influencing the performance of other closed loops Damala \& Kundu (2010) [12]. Yingwei \& Zhiyong (2011) proposed a hierarchical PLS for monitoring of penicillin process. PLS integrated with wave propagation was applied in controlling a simulated moving bed for separation of sugar and enantiomers by Junghui et al (2010). An attempt made by Minjin et al., 2007 by combining modified PLS with bias update scheme and an advanced cross validation method for inferential quality control and exhibited better performance when it was applied to industrial processes. The existence of outliers in multivariate data makes empirical modeling using univariate strategy complicated. Wang \& Srinivasan (2009) proposed a novel method that provides a robust model and retains the essential information from the data. PLS was employed to monitor the production of biodiesel from soybean (Mario et al., 2011). Whenever the operating modes of the process is often changing, monitoring and control of such process is not an easy task with multivariate statistical process monitoring techniques like PCA and PLS since these assume solely one operating point. Multiple PLS method was proposed that uses the similarity based on principal angles between the two modes to monitor the industrial pyrolysis furnace with frequently changing operating conditions (Shi et al., 2006). The application of PLS for online monitoring of chemical processes was reported by researchers like Shengiing et al. (2006), Tiina et al. (2004) and Junghuni \& Kun-Chih (2001). In this work, NNPLS based control strategies were proposed for MIMO process for set point tracking as well as disturbance rejection. 
In the identification of the MIMO distillation process, a high degree of correlation is often observed between process variables. One way to circumvent the problem is to use the PLS technique. Linear and neural network models were used to build up inner relations among the scores of the discrete input-output time series data $(X-Y)$. The $(2 \times 2)$ process was identified in latent subspaces with reasonable accuracy along with the evaluation of input-output loading matrices, which logically built up the framework for PLS \& NNPLS based process controllers. MIMO processes were casted as a series of SISO identification problems embedded in a PLS framework. Such multivariable processes could be controlled by a series of SISO controllers designed on the basis of identified dynamics in latent subspaces (transfer function relating projected $X$ i.e., $T$ and projected $Y$ i.e., $U$ ) and the PLS loading matrices (corresponding to input \& output data) being employed as pre and post compensators of the error and control signals, respectively. The rest of the paper are organized in the following way: section 2 contains a brief overview on PLS and dynamic extension of PLS; ARX and FIR model; section 3 contain identification $\&$ control of a $(2 \times 2)$ distillation process using linear dynamic PLS; section 4 contain identification \& control of the same distillation process using NNPLS; section 5 concludes and summarized the findings of this particular study.

\section{PARTIAL LEAST SQUARES}

\subsection{Linear PLS}

If two blocks of measurements say $X$ and $Y$ which are highly correlated, it becomes difficult to predict $Y$ space using only the $\mathbb{Z}$ space and the ordinary least squares technique. Several multivariate techniques such as Cannonical Correlation Analysis (CCA), Partial Least Squares (PLS), Principal component regression (PCR) have been proposed for this purpose. Input output data were generated by exciting the MIMO processes with pseudo random binary signals. $X$ and $Y$ matrices are scaled in the following way before they are processed by PLS algorithm.

$$
X=X S_{X}^{-1} \text { and } Y=Y S_{Y}^{-1}
$$

Where $S_{X}=\left[\begin{array}{cc}s_{x 1} & 0 \\ 0 & s_{x 2}\end{array}\right]$ and $S_{Y}=\left[\begin{array}{cc}s_{y 1} & 0 \\ 0 & s_{y 2}\end{array}\right]$

$S_{X}$ and $S_{Y}$ are scaled matrices. The idea of PLS is to develop a model by relating the scores of $X$ and $Y$ data. PLS model consists of outer relations that decompose $X \quad \& \quad Y$ data individually as a summation of product of score vector and loading vector and inner relations that links $X$ data to $Y$ data through their scores. The outer relationship for the input matrix and output matrix can be written as

$$
\begin{aligned}
& X=t_{1} p_{1}^{T}+t_{2} p_{2}{ }^{T}+\ldots \ldots . .+t_{n} p_{n}{ }^{T}+E=T P^{T}+E \\
& Y=u_{1} q_{1}^{T}+u_{2} q_{2}{ }^{T}+. .+u_{n} q_{n}{ }^{T}+F=U Q^{T}+F
\end{aligned}
$$

Where $T$ and $U$ represents the matrices of scores of $X$ and $Y$ while $P$ and $Q$ represent the loading matrices for $X$ and $Y$. If all the components of $X$ and $Y$ are described, the errors $E \& F$ become zero.

The inner model that relates $X$ to $Y$ is the relation between the scores $T \& U$.

$U=T B$

Where $B$ is the regression matrix. The response $Y$ can now be expressed as:

$$
Y=T B Q^{T}+F
$$

To determine the dominant direction of projection of $X$ and $Y$ data, the maximization of covariance within $X$ and $Y$ is used as a criterion. The first set of loading vectors $p_{1}$ and $q_{1}$ represent the dominant direction obtained by maximization of covariance within $X$ and $Y$. Projection of $X$ data on $p_{1}$ and $Y$ data on $q_{1}$ resulted in the first set of score vectors $t_{1}$ and $u_{1}$ , hence the establishment of outer relation. The matrices $X$ and $Y$ can now be related through their respective scores, which is called the inner model, representing a linear regression between $t_{1}$ and $u_{1}: \hat{u}_{1}=t_{1} b_{1}$. The calculation of first two dimensions is shown in Fig. 1. The residuals are calculated at this stage is given by the following equations.

$$
\begin{aligned}
& E_{1}=X-t_{1} p_{1}{ }^{\prime} \\
& F_{1}=Y-u_{1} q_{1}{ }^{\prime}=Y-t_{1} b_{1} q_{1}{ }^{\prime}
\end{aligned}
$$

The procedure for determining the scores and loading vectors is continued by using the newly computed residuals till they are small enough or the number of PLS dimensions required are exceeded. In practice, the number of PLS dimensions is calculated by percentage of variance explained and cross validation. The irrelevant directions originating from noise and redundancy are left as $E$ and $F$.

\subsection{Dynamic PLS}

For incorporation of linear dynamic relationship in a time series data in the PLS framework, the decomposition of X block is given by equation (2), the dynamic analogue of equation (3) is as follows:

$$
\begin{aligned}
& Y=G_{1}\left(t_{1}\right) q_{1}^{T}+G_{2}\left(t_{2}\right) q_{2}^{T}+\ldots . .+G_{n}\left(t_{n}\right) q_{n}^{T}+F= \\
& Y_{1}^{\exp }+Y_{2}^{\exp }+\ldots .+Y_{n}^{\exp }+F
\end{aligned}
$$

where $G_{i}$ s denote the linear dynamic model identified at each time instant by ARX model as well as FIR model and $G_{i}\left(t_{i}\right) q_{i}^{T}$ is a measure of $Y$ space explained by the $\mathrm{i}^{\text {th }}$ PLS dimension in latent subspaces. $G$ is the diagonal matrix comprising the dynamic elements identified at each of the ${ }^{\text {th }}$ latent subspaces. Figure 2 represents the PLS based dynamics prediction. Equation (9) represents the ARX structure.

$$
y(k)+a_{1} y(k-1)+a_{2} y(k-2)=b_{1} x(k-1)+b_{2} x(k-1)
$$


where $y(k)=$ output at $k^{\text {th }}$ instant, $x(k)=$ input. The input matrix for ARX based inner models used in this study was $X_{A R X}=\left\{U_{k-1}, U_{k-2}, T_{k-1}, T_{k-2}\right\}$

Finite Impulse Response Model or FIR model was also tested for inner model development. The input matrix for FIR models used: $X_{F I R}=\left\{T_{k-1}, T_{k-2}, T_{k-3}, T_{k-4}\right\}$

$T$ and $U$.represents the matrices of scores of $X$ and $Y$,respectively. The identified process transfer function:

$$
G_{P}(z)=\frac{U(z)}{T(z)}=\frac{b_{1}(z)+b_{2}(z)}{z^{2}+a_{1} z+a_{2}}
$$

The post compensation of $U$ matrix (PLS inner dynamic model output) with loading matrix $Q$ provided the PLS predicted output, $Y$. The input matrix to the PLS inner dynamic model $T$ was generated by post compensating the original $X$ matrix with loading matrix $P$. Prior to dynamic modeling, order of the model should be selected. It is difficult to choose the order of the model. Autocorrelation signals renders a good indication about order that depends on how many past input and past output values taken in the input matrix for FIR and ARX models. The model parameters for both ARX and FIR models were estimated by linear least square technique.

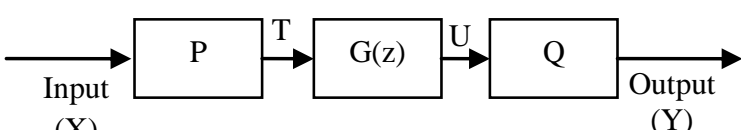

(X)

(Y)

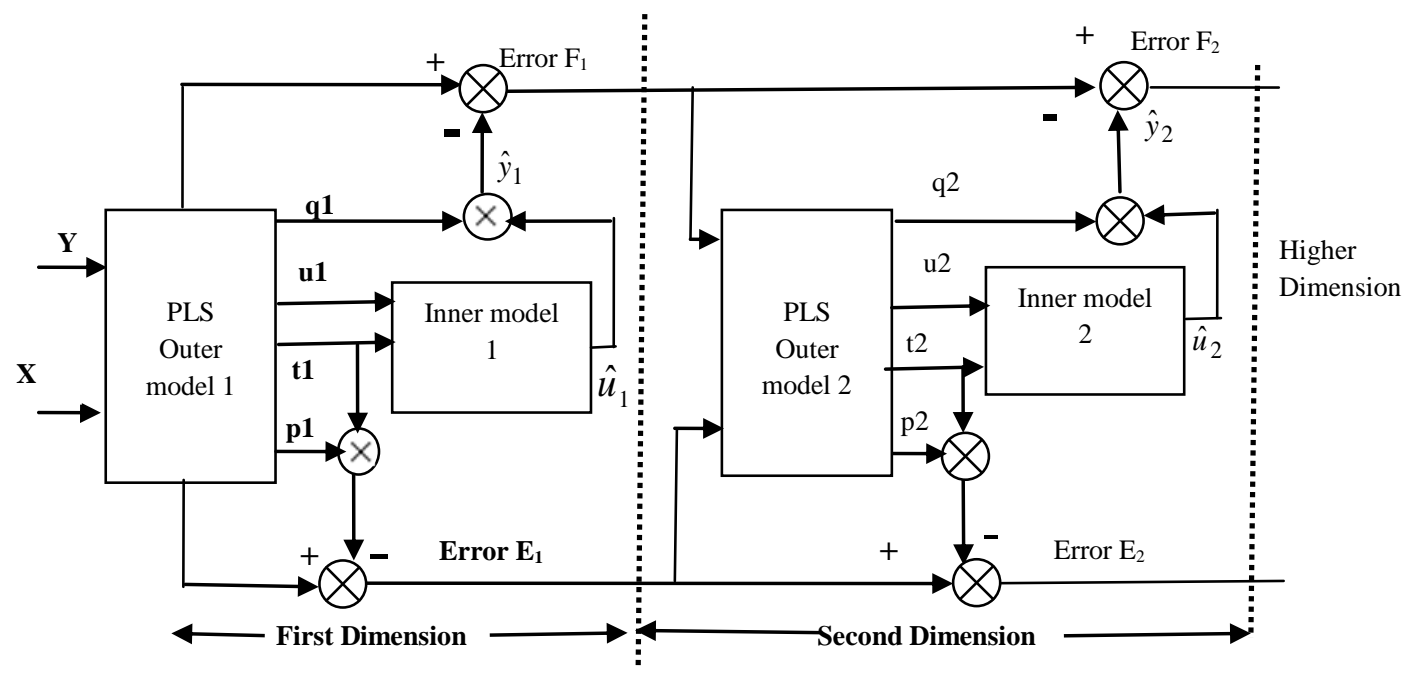

Fig 1: Standard Linear PLS Algorithm

\section{PROCESS IDENTIFICATIONA AND CONTROL SYSTEM DESIGN USING LINEAR PLS}

Series of direct synthesis SISO controllers designed on the basis of the dynamic models identified into latent subspaces were used to control the output variables in distillation column and mixing tank system being embedded in the PLS framework. The desired transfer function for closed loop simulation was selected as second order system. This approach is having two advantages; firstly, instead of using a typical multivariable controller, independent SISO controllers have been designed based on the inner dynamic model $\mathrm{G}(\mathrm{z})$ identified at each dimension $(\mathrm{G}(\mathrm{z})$ relates the scores $T$ and $U$ ) along with the error and control signals being pre and post compensated by the loading matrices namely $P$ and $Q$, respectively. Secondly, $\lambda$ is the only tuning parameter for the developed SISO controllers. The value of the tuning parameter was taken as 5. This PLSbased control strategy is presented in Figure 3. The controller was designed as direct synthesis controller in the following way,

$$
G_{C L}(s)=\left[\begin{array}{cc}
\frac{1}{(\lambda s+1)^{2}} & 0 \\
0 & \frac{1}{(\lambda s+1)^{2}}
\end{array}\right]
$$

Where $\lambda$ is tuning parameter. Direct Synthesis controller resulted is as follows: $G_{C}(s)=\frac{G_{C L}(s)}{G(s)\left(1-G_{C L}(s)\right)}$ 
$G(s)=G(z)$ represents the identified process transfer function in the latent subspaces. The controller acts on projected error $E_{u}$ i.e., actually measured output error post compensated by matrices $S_{y}^{-1} \& Q^{-1}$, respectively. $T$, the score is computed actually by the controller in closed loop PLS framework. The $T$ score then gets projected on loading matrix $P$ and transformed in to real physical inputs through $S_{x}$ which drive the processes. Because of the diagonal structure of the dynamic part of the PLS model, input-output pairings are automatic. Infeasible part of the set point vector is not allowed to pass directly to the controller because only the feasible part of set point vector is being retained after it is projected down to the latent variable subspace. This approach eliminates the futile battle which happen, generally, among the multi-loop controllers to reach an impossible target.

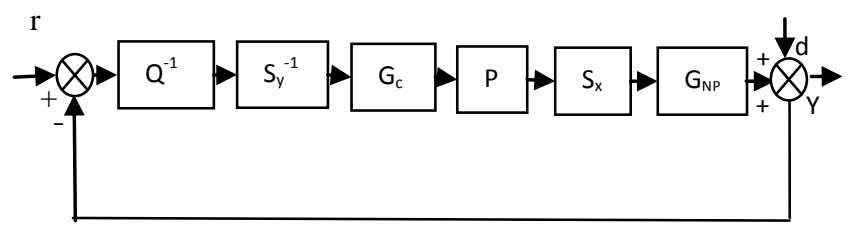

\subsection{Distillation Column}

A $2 \times 2$ distillation process was chosen to identify the process dynamics in latent subspaces and compare the PLS predicted dynamics with the actual one. Top product composition $X_{D}$ and bottom product composition $X_{B}$ were controlled by reflux rate and vapor boil-up using PLS based direct synthesis controllers. The following transfer function equation (15) was used to simulate the process when perturbed by pseudo random binary signals (1000 samples).

Inputs:

$u_{1}=$ Reflux flow rate; $u_{2}=$ vapour boil up

Disturbances:

$d_{1}=$ Feed flow rate $d_{2}=$ Feed light component mole fraction

$\left[\begin{array}{l}X_{D}(s) \\ X_{B}(s)\end{array}\right]=\left[\begin{array}{cc}\frac{0.878}{75 s+1} & \frac{-0.864}{75 s+1} \\ \frac{1.082}{75 s+1} & \frac{-1.096}{75 s+1}\end{array}\right]\left[\begin{array}{c}L(s) \\ V(s)\end{array}\right]+\left[\begin{array}{cc}\frac{0.394}{75 s+1} & \frac{0.881}{75 s+1} \\ \frac{0.586}{75 s+1} & \frac{1.119}{75 s+1}\end{array}\right]\left[\begin{array}{c}F(s) \\ Z_{F}(s)\end{array}\right]$

Both FIR based and ARX based inner models were used to identify the process dynamics in projected subspaces. Equations (16-17) represent the identified ARX based dynamic models for outputs $1 \& 2$, respectively. Equations (18-19) represent the identified FIR based dynamic models for outputs $1 \& 2$, respectively.

$G_{1}(z)=\frac{-0.001791 z-0.004398}{z^{2}+1.547 z-0.5563}$

$$
\begin{aligned}
& G_{2}(z)=\frac{-0.1367 z-0.3776}{z^{2}+1.045 z-0.05656} \\
& G_{1}(z)=\frac{0.01951 z+0.02328}{z^{2}+0.0152 z+0.0151} \\
& G_{2}(z)=\frac{0.56372 z+0.5447}{z^{2}+0.2816 z+0.5448}
\end{aligned}
$$

FIR and ARX based inner correlation between the scores $T$ and $U$ were established keeping the outer linear structure of the PLS intact. The predicted outputs corresponding to the inputs within a PLS framework were obtained by post compensating the $U$ scores with $Q$ matrix. The $T$ score were generated by post compensating the original $X$ matrix with $P$ matrix. Figures 4 and 5 present the comparison of actual plant dynamics involving top product composition $X_{D}$ and bottom product composition $X_{B}$ with ARX based PLS predicted dynamics. The performances of proposed direct synthesis controllers designed on the basis of equations (15-19) and embedded in PLS framework were examined. PLS controller perfectly could track the set point (set point change in top product composition from 0.99 to 0.996 and set point change in bottom product composition from 0.01 to 0.005). Figures 6 and 7 illustrate and compare the performance of PLS controllers ( FIR based/ARX based inner dynamic model) in servo mode.

\section{PROCESS IDEBTIFICATION AND CONTROL SYSTEM DESIGN USING NNPLS}

The process transfer function was simulated over a stipulated period of time to generate output-input data using signal to noise ratio as 10.0. The scores corresponding to all the time series data were generated using the principal component decomposition. The relationship between the $T \& U$ scores are estimated by feed forward back propagation neural network. The network input was arranged in ARX structure using the process historical database. In the closed loop simulation of the process, the inverse NN models were used as a controller typically in a feed forward fashion with the error, if any; adjusted as a bias. The inputs $\left(N_{1}\right)$ and outputs $\left(N_{3}\right)$ to the multilayer (3 layers) feed forward neural network (FFNN) representing forward dynamics of the process regarding its training $\&$ simulation phase were as follows:

Training phase

$$
\begin{aligned}
& N_{1}=\{U(t-3), U(t-2), T(t-3), T(t-2)\} \\
& N_{3}=U(t-1)
\end{aligned}
$$

Simulation Phase

$$
\begin{aligned}
& N_{1}=\{U(t-2), U(t-1), T(t-1), T(t)\} \\
& N_{3}=U(t)
\end{aligned}
$$


For DINN the simulation phase is synonymous to control phase. The number of hidden layer neurons varies from process to process. Figure 8 represents the NNPLS scheme; both in servo and regulator mode. In regulator mode, the effective disturbance transfer function was simulated to produce the $Y$ data and $D$ data; hence their corresponding scores. The FFNN representing disturbance dynamics acts as effective disturbance process. The disturbance rejection was done along with the existing servo mode.

The inputs $\left(N_{1}\right)$ and outputs $\left(N_{3}\right)$ of the multilayer (3 layers) disturbance FFNN regarding the training \& simulation phase were as follows,

Training phase

$$
\begin{aligned}
& N_{1}=\{U(t-3), U(t-2), d(t-3), d(t-2)\} \\
& N_{3}=U(t-1)
\end{aligned}
$$

Simulation Phase

$$
\begin{aligned}
& N_{1}=\{U(t-2), U(t-1), d(t-1), d(t)\} \\
& N_{3}=U(t)
\end{aligned}
$$

In the distillation process output 1 (Top Product composition, $\mathrm{X}_{\mathrm{D}}$ ) - input1 (reflux flow rate) \& the output 2 (Bottom Product composition, $\mathrm{X}_{\mathrm{B}}$ )- input 2 (vapour boilup) time series relations were identified using neural networks in a latent variable sub-space. Inverse neural network acted as controller. To control the process, 2 numbers of SISO controllers were designed. During the training phase of the NNs', the input scores to the network were arranged as per the ARX structure following eq.(20) and output or target score was set as per eq.(21). In simulation mode, the inputs and outputs to the trained networks were arranged as per eqs. (22) \& (23). Neural networks were also used to mimic the disturbance dynamics for output 1 (Top Product composition, $\mathrm{X}_{\mathrm{D}}$ ) input disturbance $1\left(\mathrm{~d}_{1}\right.$, feed flow rate $) \&$ the output 2 (Bottom Product composition, $\mathrm{X}_{\mathrm{B}}$ )- input disturbance 2 $\left(\mathrm{d}_{2}\right.$, feed light component mole fraction). During the training phase, the input scores to the network were arranged as per the ARX structure following eq. (24) and output or target score was set as per eq. (25). In simulation mode, the inputs and outputs to the trained networks were as per eqs. (26) \& (27). The training algorithm used was gradient based. The convergence criterion was MSE or mean squared error. The performances of the networks representing the process in servo and regulatory modes are presented in Table 1 .

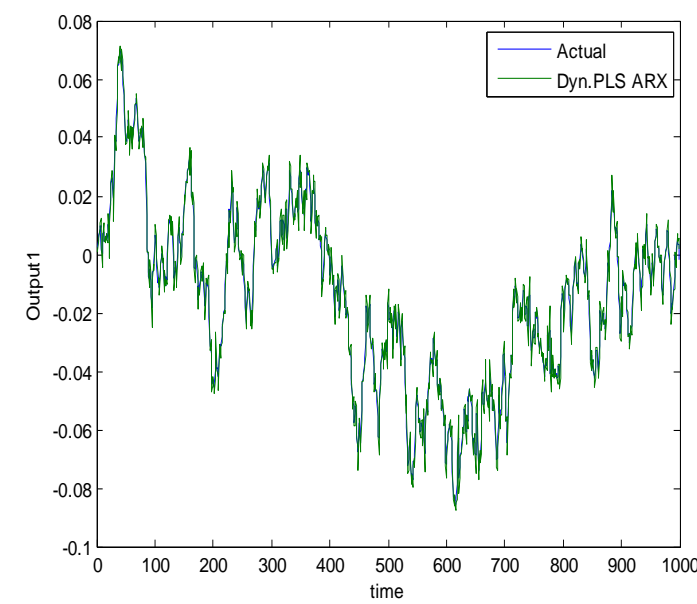

Fig 4: Comparison between actual and ARX based PLS predicted dynamics for output1 (top product composition $\left.X_{D}\right)$ in distillation process.

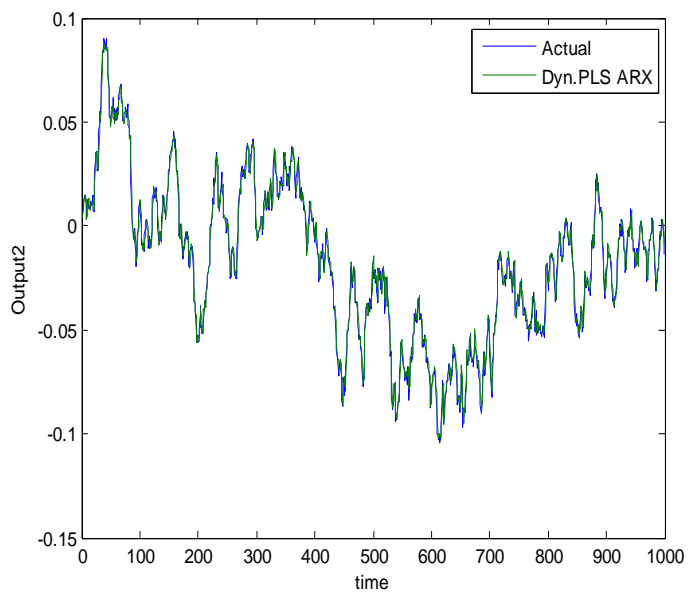

Fig 5: Comparison between actual and ARX based PLS predicted dynamics for output 2 (bottom product composition $X_{B}$ ) in distillation process.

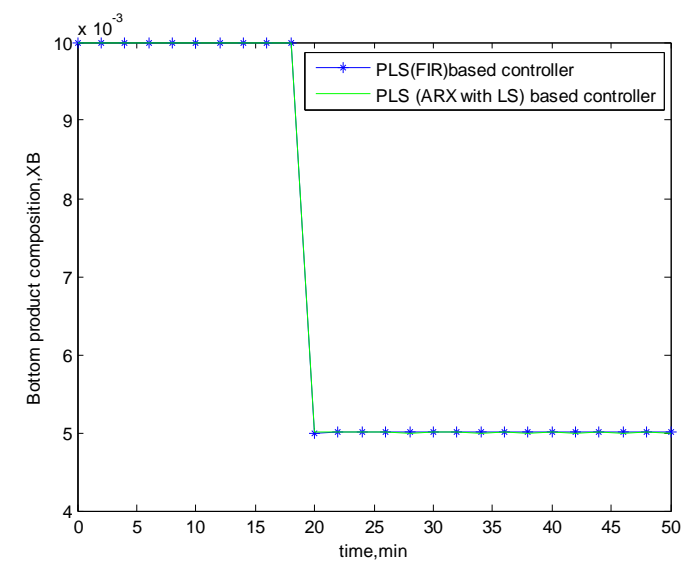

Fig 6: Comparison of the closed loop performances of ARX based and FIR PLS controllers for a set point change in $X_{D}$ from 0.99 to 0.996 . 


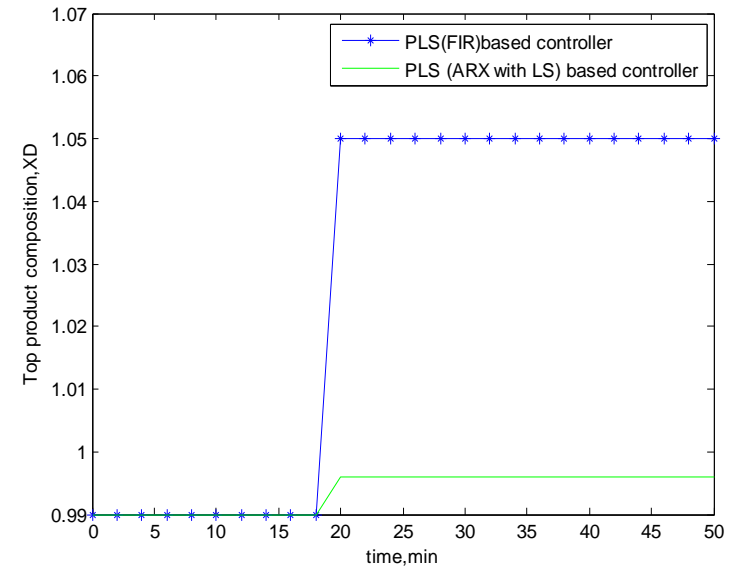

Fig 7: Comparison of the closed loop performances of ARX based and FIR PLS controllers for a set point change in $X_{B}$ from 0.01 to 0.005 .

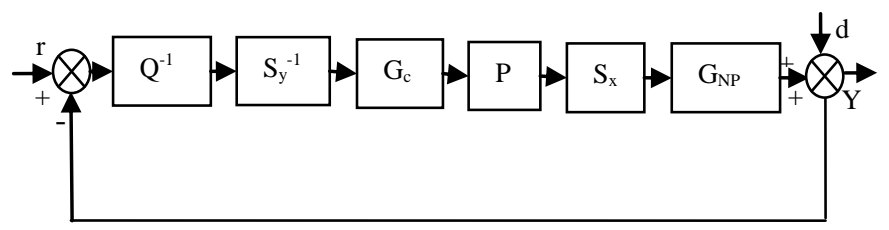

(a)

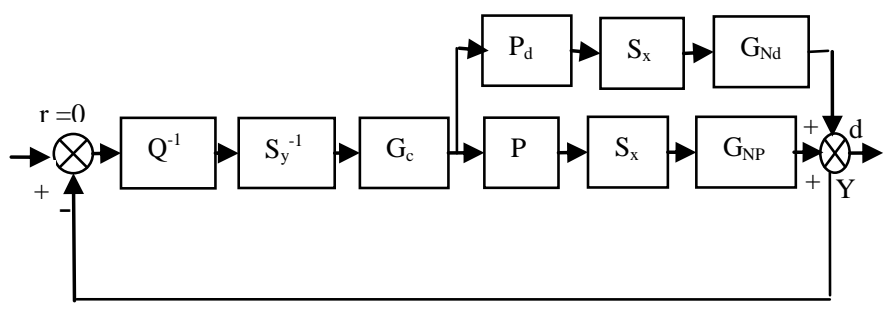

(b)

Fig 8: NNPLS control scheme (a) in servo (b) in regulator mode

Table 1. Performance criterion of the designed networks

\begin{tabular}{|c|c|c|c|c|}
\hline $\mathrm{G}(\mathrm{z})$ & $\begin{array}{c}\text { Mode of } \\
\text { Operation }\end{array}$ & $\begin{array}{c}\text { Identified } \\
\text { NNPLS }\end{array}$ & MSE & $\mathrm{R}^{2}$ \\
\hline \multirow{4}{*}{$2 \times 2$} & Servo & G1 & 0.0006807 & 0.99 \\
& & G2 & 0.0002064 & 0.99 \\
\cline { 2 - 5 } & Regulatory & Gd1 & 0.0006081 & 0.99 \\
& & Gd2 & 0.0001651 & 0.99 \\
\hline
\end{tabular}

Figure 9 presents the comparison between actual process outputs and NN identified process outputs namely the top and bottom product compositions corresponding to disturbance inputs. Figure 10 shows the closed loop performance of 2 numbers of SISO controllers. In servo mode, the controller proved to be a very reliable in set point tracking and reached the steady state value in less than $15 \mathrm{~s}$, when the top product composition changes from 0.99 to 0.996 . The $2^{\text {nd }}$ controller could track the set point changing in bottom product composition from 0.01 to 0.005 by reaching the steady state value within $10 \mathrm{~s}$. Figure 11 presents the closed loop simulation in regulatory mode in conjunction with the existing servo; showing the disturbance rejection performance in $X_{D} \& X_{B}$.
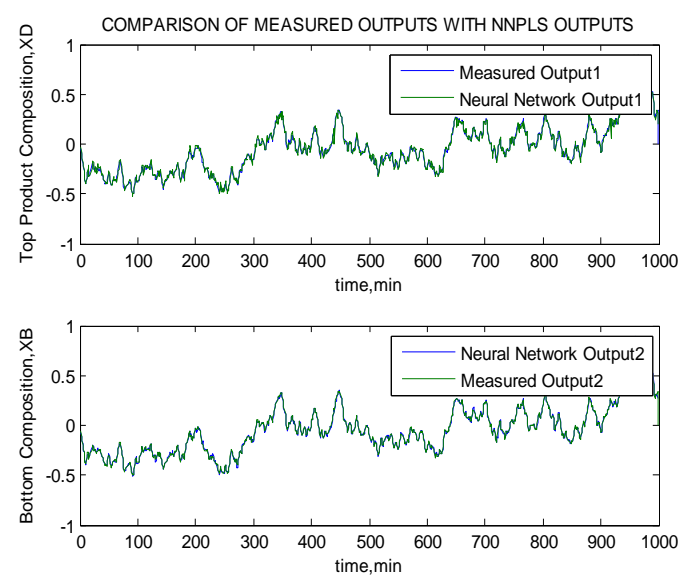

Fig 9: Comparison between actual and neutrally identified outputs of a $2 \times 2$ Distillation process in regulatory mode using projected variables in latent space.
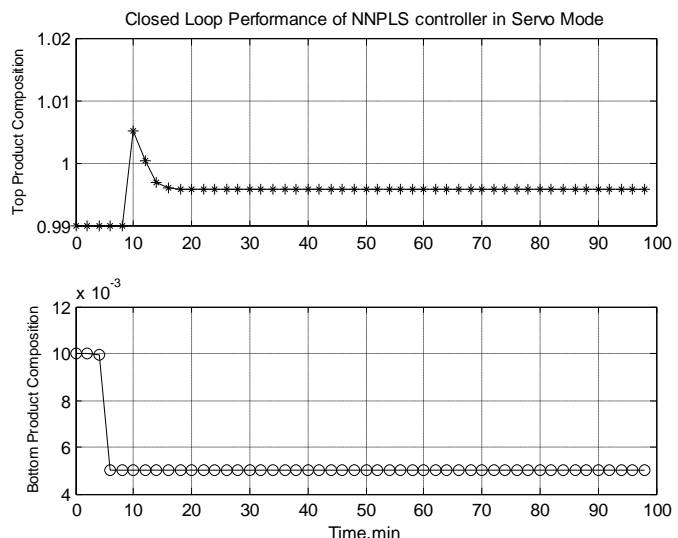

Fig 10: Closed loop response of top and bottom product composition using NNPLS control in servo mode.
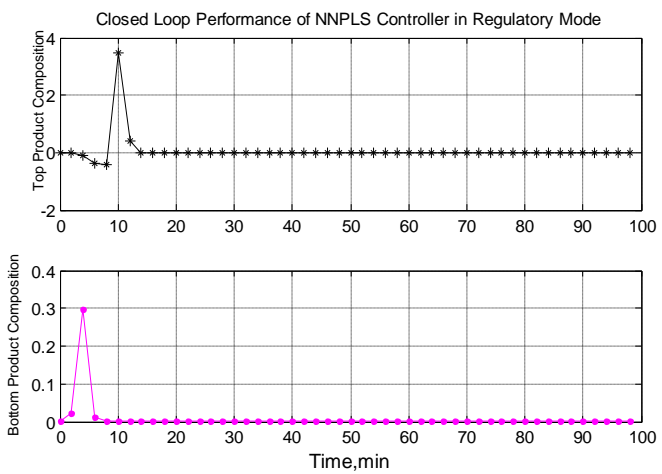

Fig 11: Closed loop response of top and bottom product composition using NNPLS control in Regulatory mode. 


\section{CONCLUSIONS}

Dynamic time series data were projected in latent subspaces. The resulted scores were correlated as the PLS inner model following ARX as well as FIR based structures. Linear least squares and neural networks were used to derive PLS inner relationship. The identified linear (both ARX and FIR based) \&NN based dynamics in the latent subspaces were used to predict the outputs corresponding to the inputs over a specified time span. In this study, the ARX-PLS predicted outputs were in much better agreement than in comparison to FIR-PLS predictions. NNPLS predicted dynamics were in excellent agreement with the actual (simulated) distillation output. Following this approach dynamic multivariable regression problems were decomposed into series of univariate regression problems. Series of direct synthesis and NN controllers designed on the basis of identified dynamics and embedded in PLS framework were used to control the distillation process considered. The performances of the PLS \& NNPLS controllers were excellent in servo as well as regulator mode.

\section{REFERENCES}

[1] H. Wold, "Estimation of principal components and related models by iterative least squares", In MultiVariate Analysis II; Krishnaiah, P. R., Ed.; Academic Press: New York, (1966), pp 391-420.

[2] P. Geladi, B. R. Kowalski, "Partial least-squares regression: A tutorial," Anal. Chim. Acta, vol. 185, (1986), pp. 1-17.

[3] S. J. Qin, T. J. McAvoy, "Nonlinear PLS modeling using neural network," Comput. Chem. Eng., vol. 16, no. 4, (1992), pp. 379-391.

[4] S. J. Qin, "A statistical perspective of neural networks for process modelling and control," In Proceedings of the 1993 Internation Symposium on Intelligent Control, Chicago, IL, (1993),pp 559-604.

[5] D. J. H. Wilson, G. W. Irwin, G. Lightbody, "Nonlinear PLS using radial basis functions," Trans. Inst. Meas. Control, vol., 19, no.4, (1997), pp. 211220.

[6] T. R. Holcomb, M. Morari, "PLS/neural networks," Comput. Chem. Eng., vol.16, no.4, (1992), pp. 393411.

[7] E. C. Malthouse, A. C. Tamhane, R. S. H. Mah, "Nonlinear partial least squares," Comput. Chem. Eng., vol. 21, no.8, (1997), pp. 875-890.

[8] S. J.Zhao, J. Zhang, Y. M. Xu, \& Z. H. Xiong, "Nonlinear projection to latent structures method and its applications", Ind. Eng. Chem. Res., vol. 45, (2006), pp.3843-3852.

[9] D. S. Lee, M.W. Lee, S. H. Woo, Y. Kim, \& J. M. Park, "Nonlinear dynamic partial least squares modeling of a full-scale biological wastewater treatment plant," Process Biochem., vol. 41, (2006), pp. 2050-2057.
[10] M. H. Kaspar, \& W. H. Ray, "Dynamic modeling for process control”, Chem. Eng. Sci., vol. 48, no. 20, (1993), pp. 3447-3467.

[11] S. Lakshminarayanan, L. Sirish, \& K. Nandakumar, "Modeling and control of multivariable processes: The dynamic projection to latent structures approach," AIChE Journal, vol. 43, (September 1997), pp. 23072323.

[12] D. Seshu kumar, M. kundu, "Design of Multivariable Neural Controllers Using A Classical Approach", IJCEA., Vol. 1, no. 2, (2010), pp. 165-172.

[13] Junghui. Chen, Kai-Ting Hsieh, Lester Lik Te, Wave propagation velocities in integrated PLS based control of a simulated moving bed process, Chem. Eng. Sci., Vol. 65 (10), (May 2010), pp. 2990-3000.

[14] Minjin Kim, In-Sun Han, Lester Lik Teck Chan, Modified PLS method for inferential quality control, Computer Aided Chemical Engineering, Vol. 15, (2003), pp. 876-881.

[15] D. Wang, R. Srinivasan, Eliminating the Effect of Multivariate Outliers in PLS based models for inferring process quality, Computer Aided Chemical Engineering, Vol. 26, (2009), pp. 755-760.

[16] Mario H. M. Killner, Jarbas J. R., Rohwedder, Celio Pasquini, A PLS regression model using NIR spectroscopy for on-line monitoring of the biodiesel production reaction, Fuel, Vol. 90 (11), (2011),pp. 3268-3273.

[17] Junghui Chen, Kai-Ting Hsieh, Lester Lik Te Chan, PLS data-driven based approach to design of a simulated moving bed process, Sep. Purif. Technol., Vol. 65 (2), (2009)

[18] Shi Jian Zhao, Jie Zhang, Yong Mao Xu, Performance monitoring of processes with multiple operating modes through multiple PLS models, J. Process Contr., Vol. 16 (7), (2006), pp. 763-772.

[19] Junghui Chen, Kun-Chih Liu, On-line batch process monitoring using dynamic PCA and dynamic PLS models, Chem. Eng. Sci., Vol. 57 (1), (2002), pp. 6375.

[20] Tiina Komulainen, Mauri Sourander, Sirkka-Lisa, Jamsa-Jounela Isa, An online application of dynamic PLS to a dearomatization process, Comput. Chem. Eng., Vol. 28 (12), (2004), pp. 2611-2619.

[21] Shengiing Mu, Yingzhi Zeng, Ruilan Liu, Ping Wu, Hongye $\mathrm{Su}$, Jian Chu, Online dual updating with recursive PLS model and its application in predicting crystal size of purified terephthalic acid (PTA) process, J. Process Contr., Vol. 16 (6), (2006), pp. 557-566. 